\title{
Das Ende der maschinellen Orthopädie
}

\author{
Von Hans Debrunner
}

Vorerst sei festgelegt, was unter maschineller Orthopädie zu verstehen ist $^{1}$.

Orthopädie beschäftigt sich mit den Leiden der menschlichen Bewegungsorgane. Ein wesentlicher Teil ihrer Grundlagenforschung liegt auf dem Gebiet der Physik, im Sektor der Mechanik. Wie die Mechanik schon im Altertum zu technischen Hilfsmitteln gegriffen hatte, um praktische Ziele zu erreichen, lag es für den Orthopäden nahe, Instrumente zu erfinden, um sich seine Arbeit zu erleichtern. Homo faber. Der Gebrauch derartiger Hilfsmittel charakterisiert die maschinelle Orthopädie. Es kann sich dabei um Instrumente handeln, mit denen der Arzt am Kranken arbeitet, z.B. Lagerungstische, Redressionsapparate, diagnostische Werkzeuge. Oder wir finden Behelfsstücke, die vom Patienten getragen werden, Schienen, Korsette, Einlagen, Ersatzglieder, die wir zusammenfassen unter dem Namen der portativen Apparate. Für die letzteren lieferte A.Schanz eine verbindliche Definition: «Im allgemeinen verstehen wir unter orthopädischen Apparaten mechanische Vorrichtungen, welche zur Behandlung von Deformitäten verwendet werden und zu deren Herstellung besondere, dem Arzt gewöhnlich nicht zur Verfügung stehende Einrichtungen gehören.» ${ }^{2}$ In der Tat wurden derartige Apparaturen zuerst von Handwerkern hergestellt, teils auf Wunsch der Ärzte, teils aus eigenem Antrieb, indem sie gleichzeitig die Krankenbehandlung übernahmen. Erst in den letzten Dezennien des 19. Jahrhunderst merkten die Orthopäden, daß ihnen damit eine unliebsame Konkurrenz entstanden war. Die Einführung der Orthopädie als Lehrfach der Medizin erleichterte es ihnen, den Anspruch auf die

1 «Machina», das für die technischen Behelfe meist gebrauchte lateinische Wort, fand im 17. Jahrhundert Eingang in die deutsche Sprache. «Dasjenige, so die Kraft vermögend machet, eine vorteilhafte Bewegung hervor zu bringen, nennt man eine Maschine» (G.A.Böckler, theatrum machinarum novum, 1673, aus Grimm, Deutsches Wörterbuch).

2 A.Schanz, Über orthopädische Apparate, Z. orthop. Chir. 4, (1899) 14. 
Führung im Apparatebau anzumelden und auch durchzusetzen. Der Aufsatz von Schanz beginnt mit den Worten: «Der unverkennbare Aufschwung, welchen die Orthopädie zur Zeit nimmt, macht sich auf dem Gebiet der Therapie hauptsächlich aus zwei Richtungen geltend: 1. in der Vervollkommnung und Ausarbeitung der operativen Methoden und 2. in der Vervollkommnung der orthopädischen Apparate.» Diese Doppelspurigkeit therapeutischer Bemühungen hat zeitweise zu einer störenden Trennung der Spezialität geführt. Es gab Ärzte, welche den blutigen Verfahren Vertrauen und Zuversicht schenkten, andere wieder, denen das konservativtechnische Vorgehen größere Erfolgsaussichten versprach. Orthopädische Chirurgie - konservative Orthopädie. Schließlich hat sich die Einsicht durchgesetzt, daß eine fruchtbare Orthopädie aus beiden Krippen zu nähren war.

In den Hippokratischen Büchern von den Knochenbrüchen und der Einrenkung der Gelenke finden sich die ersten detaillierten Angaben über maschinelle Vorrichtungen zur Erleichterung therapeutischer Maßnahmen. Man bediente sich der mechanischen Kräfte von Zug und Druck und dosierte sie durch geschickte Ausnützung der Hebelwirkung. Wo Körperkräfte nicht mehr genügten, baute man sich einfache Apparate, um gebrochene Extremitätenknochen oder luxierte Gelenke einzurichten.

Die Hippokratesübersetzungen von Emile Littré (1839-1861) und Richard Kapferer (1934-1939) enthalten Abbildungen solcher Apparate, die nach der exakten Beschreibung entworfen wurden. Sie lassen unschwer die Ähnlichkeit mit unsern noch vor kurzer Zeit verwendeten «Extensionstischen » erkennen. Sogar die Dammstütze, die im Zeitalter der Gipstechnik zur Beckenstütze entwickelt wurde, fand schon bei Hippokrates Verwendung als ein in der Mitte des Tisches eingesetzter Holzpflock, der zwischen die Beine zu liegen kam und damit dem an den Füßen angebrachten Extensionszug einen sicheren Widerhalt gab. Die Beschreibung des Modells, das im Altertum und bis über das Mittelalter hinaus unter dem Namen «Scamnum Hippocratis» benützt worden war, zeigt mit verblüffender Deutlichkeit, daß unsere späten Varianten sich kaum unterscheiden vom ehrwürdigen Vorbild. Neu an unseren Behandlungstischen ist der Einbau von metallenen Gewinden anstelle der Haspel, wodurch sich die Kraftübertragung feiner dosieren läßt, neu sind besondere Vorrichtungen zur Fixierung der kranken Körperteile; neu ist der verchromte Glanz, das Blitzblank der Instrumente, das besser in die hygienische Sauberkeit 
unserer Operationssäle paßt als das holzlederne Braun der alten Maschinen ${ }^{3}$.

Mit der Rückkehr aus dem Gestrüpp der spätscholastischen Denkweise zu den klassischen Schriften der Antike, die in der zweiten Hälfte des 15. Jahrhunderts den technischen Realismus einleitete, verstärkte sich das Interesse an seinen Problemen. Die Schriften der griechischen Mathematiker wurden erneut gelesen. In zunehmendem Maß ergriff die Lust am handwerklich-technischen Können Besitz vom Menschen. Auch die Wundärzte, insbesondere die Kriegschirurgen bemächtigten sich dieser Vorteile. Winde, Flaschenzug, Keil und Schraube wurden sozusagen wieder neu entdeckt und in der Praxis zur Bewährung gebracht. Das geschah vorerst ohne wissenschaftliche Unterlagen, auf rein empirischem Weg ${ }^{4}$.

Es ist verständlich, daß damals ein Buch zu einer gewissen Berühmtheit gelangte, das über die Vorstellungen der griechischen Medizin einen ausführlichen Aufschluß vermittelte, die Chirurgia a Graeco in Latinum conuersa wurde 1544 von dem italienischen Anatom-Chirurgen Guido Guidi herausgegeben, der am Collège de France in Paris lehrte. Dieses Kompendium enthielt ein Traktat «de machinamentis» des Oreibasios aus Pergamon, der es aus altgriechischen Quellen ausgezogen hatte. Guidis Lehrbuch war geschmückt mit Holzschnitten, die uns Bau und Verwendung solcher «machinamenta» erklären. Ein Jahr vor seiner Drucklegung 1543 - hatte Vesal sein Werk De humani corporis fabrica erscheinen lassen.

3 Rich. Kapferer, Die Werke des Hippokrates, Die Hippokratische Schriftensammlung in neuer deutscher Übersetzung, Stuttgart/Leipzig 1934-1939.

Im ersten Band des Handbuchs der orthopädischen Chirurgie von Joachimsthal (Jena 1905-1907) finden sich auf S. 1474 ff. einige Abbildungen derartiger Apparate, wie sie um 1900 von verschiedenen führenden Orthopäden benutzt und empfohlen worden waren.

4 In diesem Zusammenhang wäre das großartige Werk vom Aмвrois PARE zu erwähnen, Dix livres de Chirurgie, das nach zahlreichen Schwierigkeiten mit der Pariser Fakultät 1563 erschien. Der größte Dichter der Zeit, Pierre de Ronsard, hat dem Buch seines Freundes ein einführendes Sonett gewidmet mit den Strophen:

«Tout cela que peut faire en quarante ans d'espace

Le labeur, l'artifice et le docte savoir;

Tout cela que la main, l'usage et le devoir,

La raison et l'esprit commandent que l'on fasse,

Tu peux le voir, Lecteur, compris en peu de place

En ce livre qu'on doit pour divin recevoir;

Car c'est imiter Dieu que guérir, et pouvoir

Soulager les malheurs de notre humaine race.» 
Die Tafeln waren gezeichnet von einem Schüler Tizians, der selber sich für die Unternehmungen des Anatomen interessiert hatte ${ }^{5}$.

«Machinamenta» und «fabrica», Maschinen und Bauwerk, die Bezeichnungen entsprechen deutlich der mechanischen Betrachtungsweise der damaligen Chirurgie. Auffällig ist einzig die Tatsache, daß alle diese Apparate in ihrer Handhabung keineswegs von den Vorbildern aus Hippokrates oder Oreibasios abweichen. Man bekommt im Gegenteil den Eindruck, als hätte sich die therapeutische Phantasie vom großen Entwurf auf Kleinigkeiten zurückgezogen.

Wenn wir in Valentins Geschichte der Orthopädie die beiden Bilder der Redression eines Buckels betrachten, zwischen deren Entstehung anderthalb Jahrtausende liegen, so stellen wir mit Erstaunen fest, daß das Verfahren des Oreibasios (325-403) von Calot (1896) direkt übernommen worden ist ${ }^{6}$. Auch die vielgeschmähte «Buckelpresse» des Berliner Orthopäden Joseph ${ }^{7}$ stellt nichts anderes dar als eine moderne Auflage einer Hippokratischen Erfindung.

Die orthopädische Grundhaltung war den alten Vorstellungen treu geblieben: Es ging um die Wiederherstellung der durch Krankheit oder Verletzungen gestörten Wohlgestalt. In der Fehlform, der Deformität sah man das Wesen des Leidens, das es zu beheben galt. Die sichtbare Abweichung von der Normalgestalt wurde als Ursache der Gebrauchsstörung erkannt. Es war ein durchaus logischer Schluß zum Versuch, über die Wiederherstellung der Normalgestalt auch die Inbetriebsetzung einer normalen Funktion zu erreichen. Die gegenseitige Abhängigkeit von Form und Funktion brauchte nicht bewiesen zu werden. Man begegnete ihr auf Schritt und Tritt im handwerklichen Alltag. Ein verbogener Stab taugte nicht als Stütze; ein krummes Rad rollte mühsam mit Gerumpel. So war es augenscheinlich, daß der Klumpfüßige nicht mit dem Gesundfüßigen Schritt halten konnte. Der verbogene Stab mußte gerade geklopft, das krumme Rad gerundet, der Klumpfuß in die normale Gestalt gebracht werden.

5 Die Freundschaft zwischen Künstlern und Ärzten entsprach den Tendenzen der Zeit. Wir wissen von anatomischen Unterhaltungen zwischen Benvenuto Cellini und Guido Guidi. Leonardo stand mit Marcantonio della Torre, Michelangelo mit Matteo Colombo in Verbindung. Sie nahmen auch an Sektionen teil und nährten ihre künstlerischen Darstellungen an den wissenschaftlichen Erkenntnissen vom Bau des menschlichen Körpers.

6 Bruno Valentin, Geschichte der Orthopädie, Stuttgart 1961, S.11, Abb. 3, S.13, Abb. 5. Ein ganz ähnliches Verfahren wurde von Redard in Paris ausgearbeitet. Abb. in Schultheß (Anm. 17, S. 1467). Originalarbeit von REDARD in Presse Médicale, September 1897.

7 Joseph, Zur Streckung des Pott'schen Buckels, Berliner klin. Wschr. 37, (1901) 38. 
Dann würden sie ihren Leistungen wieder gerecht werden. So ungefähr dachte der Orthopäde im Altertum, im Mittelalter und bis zum Ausgang des 19. Jahrhunderts.

\section{II}

Die tieferen Gründe dieser Haltung, welche auch die wissenschaftliche Orthopädie des letzten Jahrhunderts bestimmte, liegen in einer allgemein materialistischen Auffassung der Natur und des Denkens. Es ging um Beschreibung und Einfangen des Geschenen in Begriffe, mit denen die Logik ihr oft bodenloses Spiel trieb. Die Wissenschaft bemühte sich, ihre innerste Überzeugung von der Durchschaubarkeit der toten und lebenden Vorgänge in allen ihren Sparten zu beweisen. «Unter dem Einfluß des wissenschaftlichen Materialismus wurde alles, was nicht mit Augen gesehen und mit Händen getastet werden konnte, zweifelhaft, ja anrüchig, weil metaphysik-verdächtig. Als wissenschaftlich und damit überhaupt als zulässig galt nur das, was entweder als materiell erkannt oder aus sinnlich wahrnehmbaren Ursachen abgeleitet werden konnte ${ }^{8}$. Im organischen Sektor war es die Gestalt, welche am ehesten dieser Denkrichtung genügte. Gestaltlehre, Morphologie, unseren Sinnen faßbar, meßbar und leicht zu beschreiben, vorzüglich im Bild nachzuvollziehen, beschäftigte die Ärzte. Der ganze Fortschritt, den die Anatomie tätigte, «lag in der Ermittelung und Präcisierung der anatomischen Thatsachen. Die Ergebnisse dieser Analyse gab die Anatomie in den Beschreibungen der Körpertheile». Formenlehre also, vertieft durch die Formvergleichung verschiedener Gattungen, immer im Hinblick auf den Menschen ${ }^{9}$.

Im Hintergrund dieser Beschreibung steht, meist unbewußt, die Auffassung vom Organismus als einer vollendet konstruierten Maschine, also

8 C. G.Jung, Die Entstehung der Seele, Gesammelte Werke, VIII, 1967.

9 Carl Gegenbauer, Lehrbuch der Anatomie, I. Band, S.31, 1903.

Es darf in diesem Zusammenhang daran erinnert werden, daß Goethes morphologische Arbeiten schon über die reine vergleichende Gestaltlehre hinaus auf die bedeutsamen Zusammenhänge mit dem Betrieblichen im lebenden Organismus weisen. Ihm ist die Gestalt «ein Bewegliches, ein Werdendes, ein Vergehendes. Gestaltenlehre ist Verwandlungslehre. Die Lehre der Metamorphose ist der Schlüssel zu allen Zeichen der Natur.» Goethes naturwissenschaftliche Schriften, Band I: Biologie, Aphorismen, Insel-Verlag, S. 652. Dazu Hans Steiner, Goethe und die vergleichende Anatomie, Gesnerus 6 (1949) 136: Die aphoristischen Aufzeichnungen Goethes bilden «eine Fundgrube origineller Gedanken und Postulate, welche teilweise erst in der modernen morphologischen Forschung Berücksichtigung und Bestätigung gefunden haben.» 
Descartes Lehre vom homo ex machina, die durch die Überlegungen der Rationalisten um Voltaire die Aufklärung beherrscht hatte. Daraus entwickelten sich die Vorstellungen von der absoluten Abhängigkeit der Funktion von der Gestalt, wie die Maschine sie tatsächlich demonstrierte. Sie kam dem gesteigerten Kausalitätsbedürfnis in erfreulichster Weise entgegen. Nicht ohne Grund hatte Delpech, der Erfinder der subkutanen Tenotomie, für unser Spezialfach den Namen «Orthomorphie» vorgeschlagen, der besser als die von Andry gewählte Bezeichnung «Orthopädie» der damaligen Auffassung entsprach ${ }^{10}$.

Im Handbuch der orthopädischen Chirurgie, das Joachimsthal 1905 herausgab, steht in der Einleitung von Riedinger zu lesen: «Obwohl sich die Orthopädie zu keiner Zeit und ausschließlich auf das Gebiet der Deformitäten beschränkt hat, so ist doch die Hauptaufgabe dieses Spezialzweiges der Chirurgie der Ausbau der Lehre von den Deformitäten, sowohl was die Pathologie als die Therapie derselben betrifft, geblieben.» Über die Pathogenese wird summarisch erklärt, «die Pathogenese der Deformitäten ist demnach in erster Linie eine mechanische ». ${ }^{11}$

Wir stehen mitten im Hochsommer materialistisch-mechanistischer Weltschau. Die Medizin hatte sich vollkommen auf die Lehren reinster Deterministik eingestellt. Absolute Präszision der Vorgänge, wie sie in der klassischen Physik durch Newton postuliert worden war, wurde auf biologische Bereiche übertragen. Die Darwinschen Lehren trugen dazu bei, im lebendigen Geschehen ebenfalls den kausal bestimmten Entwicklungsablauf zu entdecken, dem die große Weltmaschine verhaftet schien. Was lag näher, als die Manipulation des Menschen mit den Mitteln zu versuchen, welche verantwortlich gemacht wurden für den Betrieb des Weltalls? Die Technik hatte dem Arzt vielfältige Möglichkeiten geboten zur Bekämpfung der Krankheit, auf chemischem Weg, durch elektrische Impulse und rein mechanisch durch Maschinen. Gerade dieser Weg zur Beeinflussung der Formabweichung kam den Wünschen der Orthopäden entgegen. Der Glaube, daß exakte Messung und Berechnung dabei die ausschlaggebende Rolle zu spielen berufen seien, der Glaube an das Wissenschaftliche solchen

10 J.M.Delpech, a) De l'Orthomorphie, Paris 1828.

b) Die Orthomorphie oder anatomisch-pathologische Betrachtungen über die Ursachen, Vorbauungs- und Heilmittel der Hauptdeformitäten und über die wahren Grundsätze der orthopädischen Behandlung, deutsche Ausgabe, Weimar 1830.

11 Riedinger J., Wesen, Ursachen und Entstehung der Deformitäten, in Joachimsthal, Handbuch der orthopädischen Chirurgie, 1905-1907, Band 1, S.1 und 30. 
Vorgehens spiegelt sich recht naiv in den Versuchen, die orthopädischen Maschinen mit Kraftmessern, Manometern, Druckbestimmern auszustatten, die rohen Kräfte unter einem Mäntelchen zu verstecken, das die darunter getätigten Eingriffe wissenschaftlich herausputzen sollte. Das Denken in mechanischen Begriffen hatte das humane Denken überwuchert und erstickt. So ist es keineswegs verwunderlich, daß die Auswüchse der maschinellen Orthopädie um die Jahrhundertwende ihre stärksten Exuberanzen trieben.

Vielleicht wäre es dem Hallenser Anatomen Wilhelm Roux unter andern Umständen gelungen, die Gedanken der Orthopäden in eine andere Richtung zu lenken. Seine Lehre von der funktionellen Anpassung der Gewebe gewann wachsenden Einfluß auf ihre theoretische Überlegungen. Roux forderte in mahnenden Worten eine «funktionelle Orthopädie». ${ }^{12}$ Indessen gelang es ihm nicht, die Vorstellungen über die Belastungsdeformitäten zu beeinflussen, welche durch Arbeiten von Hüeter, v. Volkmann, Julius Wolff $^{13}$ begründet worden waren. Roux' Anschauungen basierten letzten Endes ebenfalls auf mechanischen Vorstellungen. Seine Lehre vom «züchtenden Kampf der Theile im Organismus» blieb Darwin und dem absoluten Determinismus verpflichtet. Die Überlegungen zur funktionellen Anpassung basierten auf mechanischen Gesetzen. Selbst die Entstehung der angeborenen Mißbildungen wurde mechanisch erklärt durch abnorme Druckverhältnisse in utero. Nur schüchtern wagten sich nach 1900 die Hinweise auf Vererbung und etwas unbestimmte innere Ursachen in die Fachblätter ${ }^{14}$. Orthopädie blieb eine mechanische Spezialität. Daß die Probleme

12 Roux Wilhelm, Gesammelte Abhandlungen über Entwicklungsmechanik der Organismen, Leipzig 1895.

Besonders wichtig der 1. Band über Funktionelle Anpassung.

13 Die Schriften von HüEteR, v. Volkmans und anderen finden in dem großen Werk von Julius WolfF, Das Gesetz der Transformation der Knochen, Berlin 1892, eine eingehende Würdigung. Doch haben sich die Wolffschen Lehren gegen die statische Auffassung der Knochenbildung nur mühsam durchsetzen können.

Siehe auch Hans Debrunner, Rich. v. Volkmann, Studien zur Darstellung von Mensch und Werk, Arch. Orthop. Chir. 31 (1932) 507-614.

14 Noch 1918 mußte Ludloff in seiner Eröffnungsrede zum XIV. Kongreß der Deutschen orthopädischen Gesellschaft in Wien seine Kollegen zu einer Revision der pathogenetischen Auffassung ermahnen. Im Hinblick auf die kongenitalen Mißbildungen sagte er: «Die bisherigen ätiologen Auffassungen können nicht mehr befriedigen ... Viele unklare Deformitäten einfach als Folgen intrauteriner Belastung erklären zu wollen, scheint mir nicht mehr berechtigt. Der Ausbau der Lehre von der Vererbung muß gefördert werden.» Verhandlungsband, S.5. 
der Rachitis, der Poliomyelitis, der Tuberkulose, diese mannigfachen Ursachen der erworbenen Mißgestaltung, später auf «chemische»Weise gelöst würden, konnte sich damals selbst der größte Optimist nicht vorstellen.

\section{III}

Die mechanische Therapie verlangte nach einem zweckmäßigen Handwerkszeug. Wir haben gesehen, daß es von den Alten übernommen wurde. Indessen verlangte der technische Fortschritt die technische Verbesserung. Neben die transportablen Zugwinden, deren sich «Schylhans» von Gersdorf als Feldchirurgus Karls des Kühnen in seinen Kriegen gegen die Eidgenossen bediente (1476-1477), neben die ebenfalls für das Sanitätsarsenal entworfene Extensionsschraube des Ambroise Paré (1564), traten im 19. Jahrhundert die als Osteoklasten bezeichneten Druckmaschinen. Robin, Trélat, Vincent in Frankreich, Oesterlen, Blasius, Heusner in Deutschland, Rizzoli in Italien, Thomas, Morton und Phelps in England erfanden derartige Apparate, mit denen schief verheilte Knochenbrüche und andere Deformitäten angegangen wurden. Bald war es soweit, daß der Orthopäde seinen wissenschaftlichen Ehrgeiz im Entwerfen von wirkungsvollen Instrumenten befriedigte. Im Lauf der Entwicklung hatte jeder Klinikleiter nach dem Vorbild Venels seinem Institut eine eigene Werkstatt angegliedert, die ursprünglich geschaffen war, um die nötigen Schienen, Stütz- und Entlastungsapparate zu bauen, aber auch die Möglichkeiten lieferte, die erfinderische Phantasie im Entwerfen neuer Redressionsmaschinen zu betätigen ${ }^{15}$.

Das steigende Tempo der Lebensführung nahm auch die Orthopädie in seinen Sog. Zeit war Geld, und Geld war Macht. Was fromme Geduld früher nach Gärtnerart durch Binden und Geradeziehn in Wochen, Monaten, Jahren zustande gebracht, das wollte man in Minuten erzwingen. Als in den Fabriken die Maschinen immer größere Dimensionen annahmen und mächtigere Kräfte freimachten, lag für den Orthopäden die Verführung nahe, es dem Maschineningenieur gleich zu tun. Die bescheiden ausgestatteten Zwingen, Zangen und Greifer wurden überboten in Größe und

15 J.A. Venel, Errichtete 1780 im waadtländischen Orbe das erste orthopädische Institut der Welt. Er ließ dort auch seine Schienen und Apparate in eigener Regie herstellen. B. Valentin, Jean André Venel, der «Vater der Orthopädie», Sudhoffs Arch. Gesch. Med. 40 (1956) 305. 
Kraft. Die «machinetta ossifraga » des Rizzoli ${ }^{16}$ vermochte trotz ihres niedlichen Namens die stärksten Knochen zu knacken. Aber die eigentlichen orthopädischen Harpyien kamen erst mit dem Aufwand an Chromstahl und Manometern. Die Apparate, welche der schwedische Instrumentenschmied Stille nach Angaben von Adolf Lorenz und anderen in Serien herstellte, waren in allen Kliniken zu finden ${ }^{17}$. Man konnte mit ihnen sorgsam und vorsichtig arbeiten; das richtete sich nach der therapeutischen Verantwortung. Ich habe die Vorteile dieser Maschinen unter meinem Lehrer Gocht schätzengelernt. Indessen ging vom Werkzeug eine teuflische Lokkung zur Ausnützung seiner Gewalten aus. Die Entscheidung über Gut und Böse entglitt oft den Händen des Arztes; er überließ sie den Kräften des Apparates. Ungeduld und Verwegenheit mißbrauchten die anvertraute Macht zum Schaden des Patienten. Wenn wir in den Tagungsberichten der deutschen Gesellschaft für Orthopädische Chirungie blättern, stoßen wir auf Reihen von Abbildungen neuer Apparaturen, raffinierter Extensionstische, minutiös ausgestatteter Osteoklasten. Ich schlage aufs Geratewohl auf: 1902 stellte Heusner einige Konstruktionen zu Extensionszwecken vor, im weiteren einen Apparat zur Beckenfixation, zur Sicherung des deformierten Fußes beim «modellierenden Redressement», für das Adolf Lorenz seinen «Osteoclastredresseur» erfunden hatte. Gocht zeigte einen Pendel- und Widerstandsapparat für Hüftleiden. 1903 meldeten sich Heusner, Fritz Lange, Wullstein, Hoeftmann, Schultze-Duisburg zum Wort, um ihre Konstruktionen vorzuführen. 1905 und 1906 wurden nicht weniger als fünf Apparate zur Skoliosenbehandlung empfohlen. Dabei begegnen wir recht eigenartigen Vorschlägen zur Gewinnung der redressierenden Kräfte. Lederriemen, Hanfschnüre, elastische Bänder und Gummizüge, Metallfedern, Schraubengewinde, das ganze Arsenal einer Mechanikerwerkstatt wurde eingesetzt und sinnreich verwendet. Machol ${ }^{18}$ brachte seinen Apparat durch strömendes Leitungswasser zur Wirkung.

16 Francesco Rizzoli, 1809-1880, dessen Name heute noch weltbekannt geblieben ist durch das von ihm gestiftete «Istituto Rizzoli» in Bologna, an dem Alessandro Codivilla und Vittorio Putti gewirkt haben, um es zu einem Zentrum der italienischen Orthopädie auszugestalten.

17 Ad.Lorenz, Heilung des Klumpfußes durch das modellierende Redressement, Wien 1895.

18 A.MAChol, Die strömende Wasserkraft im Dienste der Orthopädie, $V$. Kongreß der Deutschen Gesellschaft für Orthopädische Chirurgie, 1906, Verhandlungsband S.47. 
Aufblasbare Druckpelotten wurden eingebaut ${ }^{19}$. Fraenkel ${ }^{20}$ ging den umgekehrten Weg, indem er den Klumpfuß im Unterdruckkasten gerade zu richten versuchte. Sogar die Wasserpumpe ließ sich zu irgendwelchen Zwecken benützen. Die Erfindungslust der Orthopäden war höchstens noch zu übertreffen durch den Reichtum an phantastischen Namen, mit denen man die Maschinen versah. Vom Opomochlion über die machina aconematica, genuvalgatenda zum Dysmorphosteopalinclastes des Friedrich Oesterlen (1844) gibt es eine vergnügliche Reihe von Bezeichnungen dieser den Folterwerkzeugen nachgebildeten Maschinen ${ }^{21}$.

Alle diese Konstruktionen wurden überboten vom raumfüllenden Ungetüm, das Ferdinand Schultze-Duisburg entwickelt hatte. Mit diesem Osteoklasten ließen sich die Knochen innerhalb weniger Minuten zu Mus zusammenpressen. Ich hatte selber Gelegenheit, Schultzes Vorträge an den Tagungen der Deutschen Orthopädengesellschaft Anfang der zwanziger Jahre zu hören. Da wurde mir bewußt, wie tief man sich von den Möglichkeiten einer fast unbegrenzten Machtfülle berauschen ließ. Technik stellte sich unverhüllt als Ausdruck menschlichen Machtstrebens dar und führte sich in der Maßlosigkeit ihrer Mittel selber ad absurdum. Der Osteoklast ging an seiner Überdimensionierung zugrunde ${ }^{22}$.

Es wäre ein leichtes, die Liste zu verlängern. Doch möchte ich mich im folgenden nur mit der Skoliosentherapie befassen. In einer mustergültigen,

19 Des aufblasbaren Schlauches hat sich schon Hippokrates bedient. Im Buch über die Einrenkung der Gelenke steht zu lesen: «Ich habe einmal versucht, den Menschen auf den Rücken zu legen, einen nicht aufgeblasenen Schlauch unter den Höcker zu schieben und dann mit Hilfe eines Rohres in den untergeschobenen Schlauch Luft einzublasen. Allein damit hatte ich kein Glück ...»

«Ich habe dies absichtlich niedergeschrieben, denn gut sind auch die Erfahrungen, wo Mißlingen beim Versuch zutage trat und weshalb es mißlang. » Nach der Übersetzung von Rich. Kapferer, Abt. 22, S. 60.

20 James Fränkel, a) Redressement von Fußdeformitäten im Saugapparat, Dtsch. med. Wschr., 1908.

b) Zur Ätiologie und Therapie des angeborenen Klumpfußes, Z. orthop. Chir. 32 (1913) 115.

${ }^{21}$ In der Tat sind diese Knochenbrech- und Redressionsmaschinen nicht als durchaus eigene Erfindungen anzusehen, sondern haben ihre Vorbilder in den Folterwerkzeugen der damals üblichen Gerichtsverfahren, worauf Valentin in seiner Orthopädiegeschichte aufmerksam gemacht hat (S.143 f).

22 Ferdinand Schultze-Duisburg, Zur Behandlung der Deformitäten der unteren Extremität, Verhandlungen der Deutschen Gesellschaft für orthopädische Chirurgie, VII. Kongreß 1908, II, 1. 
heute noch lesenswerten Studie über «die Pathologie und Therapie der Rückgratsverkrümmungen ${ }^{23}$ erläuterte Wilhelm Schultheß sein Arsenal, das er aufgrund seiner profunden Kenntnisse der Skoliosenmorphologie bis ins Feinste durchdacht und spezialisiert hatte. Wir lesen vom Rumpfbeugeapparat Nr. 1 und 2, vom Schulterhebe- und vom Schulterschiebeapparat, vom Rotations- und vom Inklinationsapparat, von der Detorsionsmaschine. Die mechanischen Leistungen der Apparate bestanden in der Fixation des Patienten, in der Redression, der Applikation des Widerstandes und in der Führung der Bewegung. Man spürt, hier war ein Aufwand ingeniöser Gedanken, ein Reichtum an bastelnder Erfindungsgabe am Werk, wie sie nur zu verstehen sind aus der mechanischen Grundkonzeption der Orthopädie.

Auch aus Übersee kamen Vorschläge nach Europa. Der Umkrümmungsrahmen von Abbott fand mit seiner Technik Eingang in unsern orthopädischen Kliniken und ließ die Hoffnung auf eine befriedigende Lösung des Skoliosenproblems fast bis zur Verheißung hochschnellen ${ }^{24}$.

IV

Der Einbruch der Technik in die Turn- und Massagesäle zeitigte weitere Folgen. Die bescheidenen Requisiten, mit denen Andry und später Delpech, Jalade-Lafont, Pravaz, Jakob Heine u.a. die kindlichen Deformitäten beeinflußt hatten, Hantel, Sprossenwand, Stemmbalken, Kletterseile wurden ersetzt durch Turnmaschinen.

Die schwedische Heilgymnastik hatte aus richtigen physiologischen Überlegungen heraus den Übungswiderstand als kraftförderndes Prinzip erkannt. Die Widerstandsgymnastik ließ sich aber nicht ohne Mitwirkung eines geschulten Gymnasten durchführen. Deshalb bemühte sich Gustav Zander um eine Rationalisierung dieser Aufgaben ${ }^{25}$. Nach wissenschaftlichen Prinzipien konstruierte er entsprechende Apparate, die er durch geschickte Propaganda in aller Welt bekanntzumachen verstand. Er hatte 1865 in Stockholm ein Turninstitut mit seinen Maschinen eingerichtet. Es wurde zum Vorbild ähnlicher Anstalten von Berlin bis Buenos Aires, von Hamburg bis Baltimore. In diesen Zandersälen wurden nicht nur Kranke

23 Wilhelm Schulthess, Die Pathologie und Therapie der Rückgratsverkrümmungen, in JoachimsthaL, Handbuch der orthopädischen Chirurgie, 1905, S.487-1224.

24 Fred Aвотt, Scoliosis, Amer. J. Orthop. Surg. 1 (1917).

25 Gustav Zander, Die Apparate für mechanisch-heilgymnastische Behandlung und deren Anwendung, Stockholm 1893. 
behandelt. Das Turnen am Zanderapparat wurde zu einem Sport für ältere Damen und Herren. Es gehörte zum guten Ton, daß man sich von diesen Maschinen rütteln und schütteln ließ, daß man gegen dosierbare Widerstände ruderte, Schwungräder in Betrieb setzte und so seiner leiblichen Gesundheit einen Dienst erwies. Das «Zandern» wurde zum gesellschaftlichen Spiel und hatte seinen ursprünglichen Sinn verloren. Dafür brachte es seinem Erfinder ein Vermögen ein.

Neue Wege beschritt Hermann Krukenberg mit seinen Pendelapparaten ${ }^{26}$. Er konnte nachweisen, daß die physiologischen Überlegungen, nach denen Zander seine Vorrichtungen aufgebaut hatte, nur teilweise richtig waren. Seine Selbstbewegungsapparate arbeiten unter Ausnützung der Pendelbewegung. Sie werden durch die Eigenkraft der geschwächten Muskulatur in Gang gesetzt und durch Ausschläge der Pendel in Gang gehalten. Sie leisten bei der Bekämpfung von Kontrakturen sehr gute Dienste und werden wohl heute noch zur postoperativen Behandlung der Gelenke da und dort mit Erfolg eingesetzt.

Zu Zander und Krukenberg gesellte sich M.Herz als Erfinder auf neuen Prinzipien beruhender Widerstandsapparate ${ }^{27}$. Auch er stützte seine Überlegungen auf angeblich gesicherte Erkenntnisse der Bewegungsphysiologie, die indessen von andern bestritten wurden. In den Fachblättern lassen sich die Polemiken verfolgen, die - namentlich von Zander geschürt - gelegentlich zu bissigen Streitereien um wissenschaftliche Theorien und Prioritätsansprüche ausarteten ${ }^{28}$.

Recht umfangreich waren die Maschinen, welche zu diagnostischen Zwecken erfunden wurden. Scherbs Meridianapparat füllte ein kleines Zimmer aus. Seine Apparatur zur myokinesigraphischen Untersuchung der beim Gehen tätigen Muskulatur benötigte eine Rollgehbahn, einen Oszillographen, eine Filmkamera ${ }^{29}$. Für die Erforschung von Bewegungs-

26 Hermann Krukenberg, a) Mechanische Heilmethoden, Stuttgart 1896.

b) Mechanische Behandlung der Deformitäten, in Joachimsthal, Handbuch der orthopädischen Chirurgie, Jena 1905-1907, Band 1, S.153-208.

27 M. Herz, Neue Prinzipien und Apparate der Widerstandstherapie, Wiener med. Presse, April 1899.

28 z. B. Z. orthop. Chir. 7 (1900) 163 und a.a.O.

29 Richard ScherB

a) Über den Ablauf der Gelenkbewegungen im Allgemeinen, $\mathrm{II}^{\mathrm{e}}$ congrès de la société internationale de la Chirurgie orthopédique, 1933, S.151-188 (Untersuchungen auf der Gehrollbahn).

b) Beiheft der Z. orthop. Chir. 1920, Nr. 40 . 
abläufen und Gelenkexkursionen bleiben diese Vorrichtungen weiterhin von Nutzen.

Auch bei der Herstellung der portativen Apparate fanden die Mittel der modernen Technik Eingang. 1901 erschien die erste zusammenfassende Beschreibung der Orthopädischen Technik von Hermann Gocht ${ }^{30}$. Damit wurde zum erstenmal von ärztlicher Seite eine Anleitung geboten zur funktionsgerechten Anfertigung orthopädischer Verbandapparate. Sie erklärte die Eigenschaften der Werkstoffe und die benötigten Werkzeuge, immer im Hinblick darauf, das Handwerkliche mit den Erfordernissen des lebendigen Körpers zum Passen zu bringen. Die Herstellung exakter Gipsabgüsse und Arbeitsmodelle als Vorstufen des Apparatebaus wurden verfeinert und sorgfältig beschrieben. Indikation und Überwachung sollten vom ärztlich geschulten Verständnis ausgehen.

Bald folgten andere Schriften, welche, wie der dickleibige Band von Schanz, alles, was bisher an portativen Apparaten bekannt geworden war, mit vielen Bildern darstellten, Führer durch eine Entwicklung über Jahrhunderte hin. Aus diesen vergleichenden Beschreibungen lassen sich Fortschritte und Auswüchse im Apparatebau verfolgen ${ }^{31}$.

Ursprünglich hatten sich die Ärzte dieser Aufgaben nicht angenommen, teils aus mangelhafter Kenntnis des Arbeitsweges, teils aus gelehrter Überheblichkeit. Die Anfertigung der Apparate wurde dem Handwerk überlassen, das sich mit Geschick dieser Dinge annahm. Während die Mediziner nicht viel verstanden von den Möglichkeiten und der Ausführung maschineller Behelfsstücke zur Überwindung motorischer Ausfälle, erwarben sich umgekehrt die Apparatebauer aus praktischer Erfahrung allerhand ärztliche Kenntnisse. Sie nahmen das Recht in Anspruch, ihre Kranken selber zu behandeln, taten sich zu Gilden zusammen und beherrschten auf diese Weise ein Feld, das den Ärzten entglitten war ${ }^{32}$.

30 Hermann Gocht, Orthopädische Technik, 1. Auflage 1901, 2. Auflage umgearbeitet, Stuttgart 1917.

31 A.Schanz, Handbuch der orthopädischen Technik, 2. Auflage, Jena 1923.

32 Die Chirurgiens - Herniaires in Frankreich, die Trussmakers in England, die Orthopädisten, Bandagisten in Deutschland bildeten einträgliche Berufsverbände, die von seiten der Ärzte ganz zu unrecht der Kurpfuscherei verdächtigt wurden. Erst als sie sich selber um die Herstellung dieser Behelfsmittel zu kümmern begannen, wurde das Verhältnis zwischen Orthopädiemechaniker und Arzt zur vertrauensvollen Zusammenarbeit geleitet. Das war der Weg, auf dem die passenden Lösungen zu finden waren. Der Handwerker ist Mitarbeiter und technischer Berater des Arztes, der die Aufgaben zu stellen weiß. 
Bald erkannten einsichtige Männer, daß eine gedeihliche Entwicklung nur möglich war, wenn man die Kranken in eigenen Anstalten behandelte. Das Vorgehen des Andreas Venel, der - allerdings selber Arzt - in Orbe 1780 das welterste derartige Institut eingerichtet hatte, fand überall Nachfolge. In Deutschland waren es vor vielen andern Johann Georg Heine, der 1816 seine Anstalt in Würzburg eingerichtet hatte, sodann der Schweizer Johannes Wildberger, der in den fünfziger Jahren in Bamberg wirkte und 1856 sogar zum Ehrendoktor der Universität Jena ernannt worden war, beide nach ihrer Berufslehre Messerschmiede, oder - wie wir heute sagen würden - Mechaniker ${ }^{33}$. Vollendung brachte erst die geniale Vorstellungsgabe von Friedrich Hessing, der - ebenfalls gelernter Mechaniker - die Schienen-Hülsenapparate erfand. Damit gelang ihm die Sicherung von Fixation und Entlastung, von einwandfreiem Sitz und physiologischer Beweglichkeit, wie sie besser nicht mehr zu fordern war ${ }^{34}$.

Die Zusammenarbeit zwischen Orthopädiemechaniker und Arzt wurde mit dem Aufkommen der Orthopädie als anerkanntem medizinischem Lehrfach bewußt gefordert und weitgehend auch erreicht. Die bekannten Laienanstalten wurden der ärztlichen Leitung unterstellt. Gochts Mitteilung zur orthopädischen Technik wendet sich an den Arzt und verlangt von ihm die Vertrautheit mit den Anforderungen des Apparatebaues. Während Jahrzehnten wurden die orthopädischen Assistenten praktisch in den Klinikwerkstätten beschäftigt, um ihnen Einblick zu bieten in die handwerklichen Möglichkeiten. Was heute neu scheint, betrifft nicht das Konstruktive, sondern das Material. Mit den modernen Werkstoffen, Kunstleder, Aluminium, synthetischen Harzen und Textilien lassen sich die Apparate leichter, gefälliger und formschöner herstellen. Doch läßt sich in diesem Zweig der maschinellen Orthopädie seither ein deutlicher Verwendungsrückgang feststellen. Das «Los vom Apparat» ist zu einem

${ }^{33} \mathrm{Zu}$ Joh. Georg Heine, siehe Anmerkung 5, S.223 f. Zu Wildberger siehe Gerhard Grosch, Johannes Wildberger, ein Schweizer Messerschmied und Wegbereiter der Orthopädie, Basler Veröffentlichung zur Geschichte der Medizin und Biologie, Fasc. XXVII, Basel 1969.

34 Friedrich Hessing, (1838-1918), hatte seine Anstalt in Göggingen bei Augsburg eingerichtet. Sie wurde dank den vorzüglichen Apparaten weltbekannt. Sie bedeuteten einen großen Fortschritt in der orthopädischen Mechanik. Die Anpassung an die knöchernen Formen des Körpers war eine viel genauere als bei den bisher gebrauchten Modellen. Das verwendete Material, Gewebe, Leder und Stahlgerüst war so angeordnet, daß der Verstellbarkeit der Apparate großer Spielraum geöffnet war. 
orthopädischen Slogan geworden, dem die operative Therapie in vielen Fällen entsprechen kann. So sehen wir denn am Anfang des 20. Jahrhunderts die maschinelle Orthopädie ihrem Ende entgegengehen. Die geistreich ersonnenen Maschinen rosten in den Kellern der Hospitäler oder sind als Schrott anderen Zwecken zugeschmolzen.

Auch die Modelle zur Skoliosenbehandlung, welche den Instituten einst ihre Bedeutung verschafft hatten, büßten sie ein, teils weil die Ergebnisse nicht befriedigten, teils weil mit dem Tode ihrer Erfinder der Glaube an ihre Zweckmäßigkeit verlorengegangen war. Die Pendel- und Widerstandsmaschinen wurden aus den Turnsälen fortgeschafft, in deren freiem Raum heute wieder mit den einfachen Requisiten der vormaschinellen Zeit gearbeitet wird.

Hinter diesen Vorgängen, die überall ungefähr gleichzeitig einsetzten und in den dreißiger Jahren beendet waren, stehen verschiedene entwicklungstreibende Kräfte. Sie sind vor allem zu suchen in einer radikalen Umstellung der Denkweise, kommen also aus Quellen, welche die gesamte Zivilisation betreffen. Der technische Materialismus neigt zur Perfektion, wird also zweifellos vom Bestreben geleitet, einen bestmöglichen Endzustand zu erreichen, sich statisch auszurichten auf ein bleibendes Muster der Vollkommenheit. Die neuen Erkenntnisse der Naturwissenschaften weisen andere Bilder. Anstelle der klassischen Ausgewogenheit, wie sie uns noch verkündet wird in Büchners Kraft und Stoff, in Haeckels Welträtseln, einst Bestseller und Leitbilder der materialistischen Weltanschauung ${ }^{35}$, ist ein Dynamismus getreten, der auch die Biologie und damit die Medizin ergriff. «Um die Jahrhundertwende wußte der Physiker, daß die mechanistische Weltanschauung aufgegeben werden muß.» ${ }^{36}$ Neben die Mechanik traten Elektrodynamik und Thermodynamik als ebenbürtige wissenschaftliche Forschungsgebiete. Dynamik also, wie sie denn auch die Chemie bot, die im Vergleich zur Physik eh und je etwas Lebendiges vertreten hatte. Sie bewegte die Gedanken der Biologen. Dynamik stellt sich im lebenden Organismus sozusagen in Reinheit dar. Erbvorgänge traten

35 Haeckels Schrift, 1899 erschienen, wendet sich an den gebildeten Laien und gilt als Hauptstütze des späten Materialismus der zweiten Hälfte des vorigen Jahrhunderts. BüchNer gab sein Opus 1855 in Druck und bereitete damit dem krassesten Materialismus Zugang zu den Köpfen vieler fortschrittsgläubiger Zeitgenossen. Von der Sprachgewalt und der dichterischen Erkenntnis seines Bruders Georg (Dantons Tod, Wozzek) spürt man bei Ludwig keinen Hauch.

${ }^{36}$ Horst Hiller, Raum, Zeit, Materie, Unendlichkeit, Stuttgart 1964, S. 158. 
anstelle der mechanischen Ursachen angeborener Mißbildungen. Dem Wachstum wurden gestaltende Einflüsse im rechten und im falschen zugebilligt und für therapeutische Zwecke ausgenützt. Die Vorstellungen vom Ablauf des lebendigen Geschehens verdrängten allmählich die $\mathrm{Zu}$ standsbilder, auf die der Krankheitsbegriff zurückgeführt worden war. Nicht mehr die Fehlform faszinierte die orthopädische Anteilnahme. Als das Wesentliche der Störung drängt sich der Fehlgang des Bewegungsorganes ins Interessefeld. Die Korrektur der Deformität als bloße Umgestaltung zur normalen Form stand nicht mehr im Zentrum orthopädischen Heilstrebens. Die Verbesserung der gestörten Funktion wurde Ziel seiner Bemühungen. Fehlleistungen lassen sich nicht mit Maschinen angehen. Eine Verlegung der therapeutischen Maßnahmen von außen ins Körperinnere durch operative Eingriffe an den Kraftspendern der Motorik, durch Umstellung am Skelett versuchte die Eigenkräfte des Körpers zu mobilisieren. Bekanntlich wurden über solche Gedankengänge erfreuliche Erfolge gewonnen (Sehnenverlagerungen, Nervenoperationen, Gelenkplastiken, Wachstumsbeeinflussung, operative Bekämpfung von Entzündungsherden und anderes mehr).

Die Technik des geradlinigen Fortschrittes zu einem bleibenden Ziel, zu einer einmaligen Wahrheit hat sich verwandelt in eine Technik dynamischer Unruhe, die nicht mehr die Stille abgeschlossener Erkenntnis anpeilt, sondern sich auf verschiedensten Sektoren unseres Forschens in die Breite bewegt. Wir sind der Dynamik verfallen und damit der menschlichen Kühnheit. Auch der Arzt dringt in Gebiete vor, die voller Unruhe, voller Gefahren sind, und sein Denken weist ihm den Fortschritt als ständiges Fortschreiten ins Nie-Abgeschlossene. Das wird zur Ursache eines Vertrauens auf die dynamischen Kräfte in der Biologie. Die ganze Heilkunde wird funktionell ausgerichtet. «Medizin in Bewegung»(Siebeck). Der menschliche Muskel kann mehr als der Knochen. Wenn auch der Knochen unentbehrlich ist für die Statik des Gerüstes, so macht erst die vom Muskel gesteuerte Motorik den orthopädisch interessanten Menschen. Der Auszug aus dem Maschinensaal passiver Betätigung zum Sportplatz, zur Heilgymnastik, zur aktiven Selbsthilfe wird erklärlich.

Die gleichen Gründe haben dazu geführt, den Kranken mit immer kühnerem Zugriff durch die Operation von seinen Beschwerden zu befreien. Die Operation ist anstelle der Redression getreten und hat das plumpe Behandlungsrüstzeug durch geschliffene Klingen und blitzende Instrumente ersetzt. Sie bringt die nötigen Gewebsverletzungen unter 
Sichtkontrolle. Die gefürchteten Gefahren - Blutungen, Infektion, Embolie - werden von Jahr zu Jahr geringer, die Lust an der operativen Methodik und das Vertrauen auf ihre Leistungen von Jahr zu Jahr größer ${ }^{37}$.

Die Orthopädie hat sich aus ihrer einseitig mechanischen Sonderung zurückgefunden zu den Methoden der allgemeinen Medizin. Sie sieht nicht nur verbogene Gliedmassen, sie sieht das erkrankte Glied, den an der Erkrankung seiner Motorik leidenden Menschen. Damit hat sie sich aus dem Arsenal von Apparaturen und Maschinen wieder freigemacht für den $\mathrm{Zu}-$ gang zu dem Schatz allgemeiner Heilmethoden, den die Medizin in den letzten Dekaden geäufnet hat.

So verstehe ich die Tatsache, daß die maschinelle Orthopädie innerhalb weniger Jahre zum Verschwinden kam. Sogar der Gipsverband, seit über hundert Jahren segensreich für die Behandlung der Frakturen, scheinbar unentbehrliches Requisit der orthopädischen Therapie, verliert seine Bedeutung als Fixativ. Er weicht der inneren Drucksicherung durch Schrauben und Metallschienen. Und wiederum sind es funktionelle Überlegungen, welche die Vorteile früher Bewegungsmöglichkeit gegenüber der langen Ruhigstellung der Gelenke im Gips betonen.

Sind wir nun wirklich berechtigt, vom Ende der maschinellen Orthopädie zu sprechen ? Oder stellt sich dieser Schluß doch als Täuschung heraus? Eine neue Art der Verwendung maschineller Vorrichtungen beginnt sich durchzusetzen, deren Möglichkeiten wir noch nicht übersehen. Tausende von Menschen verdanken ihre wiedergewonnene Aktivität den künstlichen Hüftköpfen. Ganze Gelenke werden durch die sogenannten Totalprothesen, also durch richtige kleine Maschinen ersetzt. Frakturen werden durch angelagerte Metallschienen verschraubt und mittels ingeniöser Vorrichtungen unter kallusfördernden Druck gesetzt. Die Technik läßt sich scheinbar nicht aus der Orthopädie verdrängen: sie war zu allen Zeiten von ihrem Geist durchdrungen.

Ich denke an ein Wort von Claude Bernard: «L'homme peut plus qu'il ne sait.» Es weist hin auf die Gefahren des wissenlosen Handelns. Möge es uns bewahren vor der gewissenlosen Tat!

37 Die damaligen orthopädisch tätigen Chirurgen wirkten alle im mittleren und späteren 19. Jahrhundert. Bewußt hat erst Nicoladoni das Prinzip der primären Korrektur der Fehlform verlassen, als er in den achtziger Jahren mit seiner Sehnentransplantation den normalen Gewebeverband veränderte, um funktionelle Besserung zu erreichen. 